\title{
A boundary element solution to the problem of interacting a.c. fields in parallel conductors
}

\author{
EINAR M. RØNQUIST $\dagger$ and TERJE SIRA $\dagger$
}

\begin{abstract}
The a.c. fields in electrically insulated conductors will interact through the surrounding electromagnetic fields. The pertinent field equations reduce to the Helmholtz equation inside each conductor (interior problem), and to the Laplace equation outside the conductors (exterior problem). These equations are transformed to integral equations, with the magnetic vector potential and its normal derivative on the boundaries as unknowns. The integral equations are then approximated by sets of algebraic equations. The interior problem involves only unknowns on the boundary of each conductor, while the exterior problem couples unknowns from several conductors. The interior and the exterior problem are coupled through the field continuity conditions. The full set of equations is solved by standard Gaussian elimination. We also show how the total current and the dissipated power within each conductor can be expressed as boundary integrals. Finally, computational results for a sample problem are compared with a finite difference solution.
\end{abstract}

\section{Introduction}

To solve skin effect and eddy current problems involving slowly time-varying fields, the quasi-stationary approximation to Maxwell's equations is applied. The assumptions are that the displacement currents can be ignored compared with the conduction currents.

Both two-dimensional (Chari 1973, Andersen 1977, Salon et al. 1981) and full three-dimensional problems (Carpenter 1977, Ekrann and Sira 1982) have been studied. Most authors use finite difference or finite element solution techniques.

Salon et al. (1981) present a boundary element solution to the eddy current problem by using a Helmholtz type equation for the electric vector potential. The magnetic field is in this case unidirectional, and perpendicular to the two-dimensional, finite domain in which the calculations are made.

Andersen (1977) presents a finite element solution to two-dimensional skin effect and eddy current problems. The governing field equations are expressed by the magnetic vector potential in combination with the electric scalar potential.

In our paper, we shall apply the latter formulation in solving the problem of interacting a.c. fields in electrically insulated, parallel conductors. We present a boundary element solution to the two-dimensional problem with an infinite domain partitioned into subdomains. The media is linear and isotropic with constant electric conductivity and magnetic permeability within each conductor. The total current in each conductor is also assumed to be known. The governing field equations are transformed to boundary integral equations and then solved. The total current and the dissipated

Received 26 September 1983.

$\dagger$ Institute for Energy Technology, Kjeller, Norway.

M.I.C. 
power within each conductor are usually expressed as integrals over the volume. We show how these can be transformed into integrals involving only the unknowns on the boundary.

\section{Mathematical formulation}

The electric field $\boldsymbol{E}$ and the magnetic field $\boldsymbol{H}$ can be expressed by the magnetic vector potential $A$ and the electric scalar potential $V$

$$
\begin{aligned}
& \boldsymbol{E}=-\frac{\partial A}{\partial t}-\nabla V \\
& \boldsymbol{H}=\frac{1}{\mu} \nabla \times \boldsymbol{A}
\end{aligned}
$$

Using the gauge $\nabla \cdot A=0$ and Maxwell's equations, we get

$$
\nabla^{2} A=-\mu J
$$

where

$$
\boldsymbol{J}=\boldsymbol{\sigma} E
$$

We want to find equations for $A$ inside a conductor $(R)$ and in the surrounding region $\left(R^{0}\right)$, see Fig. 1.

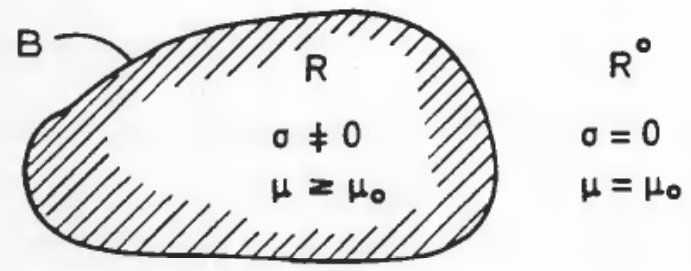

Figure 1. Cross-section $R$ of a conductor surrounded by a non-conducting, non-magnetic region $R^{0}$.

In our two-dimensional problem, $A, J, E$ and $\nabla V$ are all perpendicular to the inplane magnetic field. For that reason, we shall drop the vector notation. Assuming steady-state, sinusoidal conditions and using complex notation, (1), (3) and (4) give us the Helmholtz equation for $A$ inside region $R$

$$
\nabla^{2} A-\alpha^{2} A=-\alpha^{2} \phi
$$

where

$$
\begin{aligned}
& \alpha^{2}=i \omega \mu \sigma \\
& \phi=-\nabla V / i \omega
\end{aligned}
$$

In the region $R^{0}$ surrounding the conductor, $\sigma=0$ and the Helmholtz equation reduces to the Laplace equation

$$
\nabla^{2} A=0
$$


The tangential component of the magnetic field is continuous across an interface between two different media. Boundary conditions for the magnetic vector potential across the interface $B$ between $R$ and $R^{0}$ are therefore given as

(i) $A$ is continuous

(ii) $\frac{1}{\mu} \frac{\partial A}{\partial n}$ is continuous

Using (1), (4) and (7), we express $E$ and $J$ in terms of $A$ and $\phi$

$$
\begin{aligned}
& E=-i \omega(A-\phi) \\
& J=-i \omega \sigma(A-\phi)
\end{aligned}
$$

We are only interested in the current distribution inside the conductor. Hence, it is not necessary to know $\phi$ on the outside. Since $\phi$ is a unidirectional, curl free vector field, $\phi$ is a constant within the conductor. This constant can be found from the known total current $\boldsymbol{I}$ flowing in the conductor. Integrating the current density $\boldsymbol{J}$ over a crosssection, we get

$$
I=\int_{R} J d R
$$

We are also interested in the dissipated power $P$ inside the conductor. We can write $P$ as

where

$$
P=\int_{R} w d R
$$

$$
w=\sigma|E|^{2}
$$

\section{Boundary integral formulation}

In this section, we express the Helmholtz equation and the Laplace equation in terms of boundary integrals. We also express the dissipated power $\boldsymbol{P}$ and the total current $I$ flowing in each conductor as boundary integrals.

The Green function $G_{H}$ for the Helmholtz equation is found by solving

$$
\nabla^{2} G_{H}-\alpha^{2} G_{H}=\delta\left(r-r^{\prime}\right)
$$

In two dimensions, $G_{H}$ is given as

where

$$
G_{H}=-\frac{1}{2 \pi} K_{0}(\alpha r)
$$

$$
\boldsymbol{r}=\left|\boldsymbol{r}-\boldsymbol{r}^{\prime}\right|
$$

In (14), $\boldsymbol{r}$ and $\boldsymbol{r}^{\prime}$ are the field and source points respectively. The operator $\nabla^{2}$ acts on the field point $r . K_{0}$ is the modified Bessel function of the second kind, order zero. Using standard technique, the magnetic vector potential at a point $r \in R$ is written as

$$
A(\boldsymbol{r})=-\alpha^{2} \phi \int_{R} G_{H} d R+\int_{B}\left(A \frac{\partial G_{H}}{\partial n}-G_{H} \frac{\partial A}{\partial n}\right) d B
$$

where $\partial / \partial n$ denotes the normal derivative out of the conductor (region $R$ ). Integrating (14) over the region $R$ and using the divergence theorem, we get

$$
A(r)=\left(1-\int_{B} \frac{\partial G_{H}}{\partial n} d B\right) \phi+\int_{B}\left(A \frac{\partial G_{H}}{\partial n}-G_{H} \frac{\partial A}{\partial n}\right) d B
$$

M.r.c. 
Inserting for $G_{H}$ and $\partial G_{H} / \partial n$ where

$$
\frac{\partial G_{H}}{\partial n}=\frac{\partial G_{H}}{\partial r} \frac{\partial r}{\partial n}=\frac{\alpha}{2 \pi} K_{1}(\alpha r) \cos \psi
$$

eqn. (18) is written as

$$
\begin{aligned}
A(\boldsymbol{r})=\left(1-\frac{\alpha}{2 \pi} \int_{B} K_{1}(\alpha r) \cos \psi d B\right) \phi+\frac{\alpha}{2 \pi} \int_{B} A K_{1}(\alpha r) \cos \psi d B & \\
& +\frac{1}{2 \pi} \int_{B} \frac{\partial A}{\partial n} K_{0}(\alpha r) d B
\end{aligned}
$$

where $K_{1}$ is the modified Bessel function of the second kind, order one, and $\cos \psi$ is the direction cosine.

When $r$ is a point $r_{B}$ on the boundary $B$, we use the same method as in Salon et al. (1981) to remove the singularities.

$$
\begin{aligned}
A\left(r_{B}\right)=\left(1-\frac{\alpha}{\pi} \int_{B} K_{1}(\alpha r) \cos \psi d B\right) \phi+\frac{\alpha}{\pi} \int_{B} A K_{1}(\alpha r) \cos \psi d B & \\
& +\frac{1}{\pi} \int_{B} \frac{\partial A}{\partial n} K_{0}(\alpha r) d B
\end{aligned}
$$

Correspondingly, for the Laplace equation, the Green function $G_{L}$ satisfies

$$
\nabla^{2} G_{L}=\delta\left(\boldsymbol{r}-\boldsymbol{r}^{\prime}\right)
$$

and has the two-dimensional solution

$$
G_{L}=\frac{1}{2 \pi} \ln r
$$

The magnetic vector potential at a point $r \in R^{0}$ outside the conductor can then be written as

$$
A(\boldsymbol{r})=\int_{B^{0}}\left(A \frac{\partial G_{L}}{\partial n^{0}}-G_{L} \frac{\partial A}{\partial n^{0}}\right) d B
$$

where $\partial / \partial n^{0}$ denotes the normal derivative out of region $R^{0}$ with boundary $B^{0}$.

In Fig. 1, where there is only one conductor, the boundary $B^{0}$ consists of the boundary $B$ plus a boundary $B_{\infty}$ at infinity. The contribution of the integral from $B_{\infty}$ will be zero, so we can put $B^{0}=B$. We also have $\partial / \partial n^{0}=-\partial / \partial n$.

When $r$ is a point $r_{B}$ on the boundary $B^{0}$, we get, after removing the singularities,

$$
A\left(\boldsymbol{r}_{B}\right)=\frac{1}{\pi} \int_{B^{\circ}}\left(A \frac{\cos \psi^{0}}{r}-\frac{\partial A}{\partial n^{0}} \ln r\right) d B
$$

Since $J$ can be expressed as a divergence by (3), the total current $I$ can also be written as a boundary integral. Using (11), (3) and the divergence theorem, the total current $I$ is written as

$$
I=-\frac{1}{\mu} \int_{B} \frac{\partial A}{\partial n} d B
$$


If we can express $w$ in eqn. (12) as a divergence, we can also write the dissipated power $P$ as a boundary integral. We define the vector $S$ directed in the plane as

$$
S=E \times H^{*}
$$

where ${ }^{*}$ denotes the complex conjugate. Taking the divergence on both sides of eqn. (27) and using Maxwell's equations, we get

$$
w=-\operatorname{Re} \nabla \cdot S
$$

Inserting (27) and (28) in eqn. (12) and using the divergence theorem, we write $P$ as

$$
P=-\operatorname{Re} \int_{B} E H_{\|}^{*} d B
$$

where $H_{\|}$is the tangential component of the magnetic field along the boundary $B$. Using (2), $H_{\|}$can be written as

$$
H_{\mathrm{H}}=-\frac{1}{\mu} \frac{\partial A}{\partial n}
$$

Inserting for $E$ and $H_{\|}$from (9) and (30), we get

$$
P=-\frac{\omega}{\mu} \operatorname{Re} \int_{B} i(A-\phi) \frac{\partial A^{*}}{\partial n} d B
$$

If the current distribution can be approximated with a line current $I$ at a point $r_{L}$, we can evaluate the integral in (24). Let $B_{\varepsilon}$ be the boundary of region $R_{\varepsilon}$, a circular, conducting region with radius $\varepsilon$ placed at $r_{L}$. Using (24), (26) and the continuity relation (ii), we get

$$
A(r)=\lim _{\varepsilon \rightarrow 0} \int_{B_{\varepsilon}}\left(A \frac{\partial G_{L}}{\partial n^{0}}-G_{L} \frac{\partial A}{\partial n^{0}}\right) d B=-\frac{\mu_{0} I}{2 \pi} \ln \left|r-r_{L}\right|
$$

for a point $\boldsymbol{r} \in R^{0}$.

\section{Numerical formulation}

We now turn our attention to the problem of computing $A, \partial A / \partial n$ and $\phi$ in the more general case involving several conductors. We approximate the boundary integral equations (21), (25) and (26) by a set of algebraic equations.

We shall use the following notation, see Fig. 2. The subscript $m$ refers to conductor no. $m$. We have $M$ conducting regions $R_{m}$ with boundaries $B_{m}$ (in Fig. 2, $B_{3}=B_{3}{ }^{-} \cup B_{3}{ }^{+}$), and $K$ non-conducting, non-magnetic regions $R_{k}{ }^{0}$ with boundaries $B_{k}{ }^{0}$. A boundary $B_{k}{ }^{0}$ will always be a part of the total boundary $B=B_{1} \cup \ldots \cup B_{m} \cup \ldots$

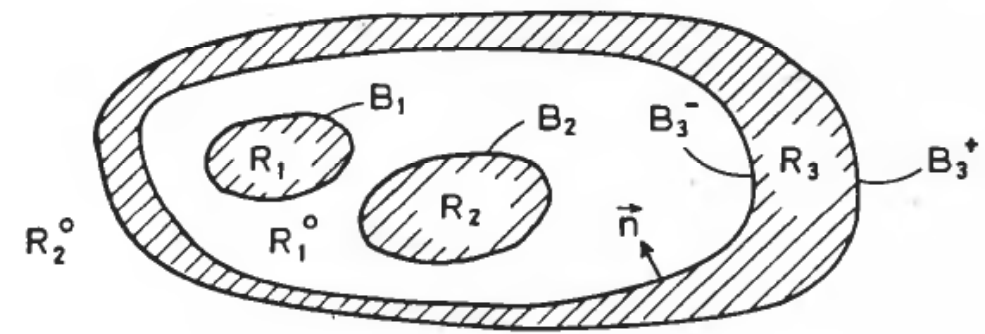

Figure 2. 
$\cup B_{M}$. For example, the boundary $B_{1}{ }^{0}$ of region $R_{1}{ }^{0}$ in Fig. 2 is $B_{1}{ }^{0}=B_{1} \cup B_{2} \cup B_{3}{ }^{-}$ while the boundary $B_{2}{ }^{0}$ of region $R_{2}{ }^{0}$ is $B_{2}{ }^{0}=B_{3}{ }^{+}$(the boundary at infinity does not contribute to the integral). The normal derivative out of region $R_{m}$ and $R_{k}{ }^{0}$ is denoted by $\partial / \partial n$ and $\partial / \partial n^{0}$ respectively.

We approximate the boundary $B$ by $N$ straight line elements $\Delta l_{i}, i=1, \ldots, N$. The values of the magnetic vector potential $\left(A_{i}, A_{i}{ }^{0}\right)$ and its normal derivative $\left(\partial A_{i} / \partial n, \partial A_{i}{ }^{0} / \partial n^{0}\right)$ on the two sides of an element $\Delta l_{i}$ are assumed to be constant along $\Delta l_{i}$ and equal to their values in the mid-point of the element.

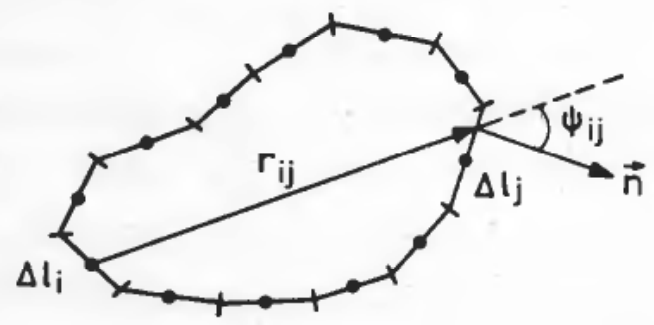

Figure 3. Approximating the boundary with straight line elements.

Using the notation as shown in Fig. 3, we approximate (21) as

$$
A_{t}-\left(1-\sum_{j \neq i} a_{i j}\right) \phi_{m}+\sum_{i \neq i} a_{i j} A_{j}+\sum_{j} b_{i j} \frac{\partial A_{j}}{\partial n}
$$

where $i, j \in B_{m}$ and

$$
\begin{gathered}
a_{i j}=\frac{\alpha}{\pi} \int_{\Delta l_{j}} K_{1}\left(\alpha r_{i j}\right) \cos \psi_{i j} d l \\
b_{i j}=\frac{1}{\pi_{\Delta l_{j}}} K_{0}\left(\alpha r_{i j}\right) d l
\end{gathered}
$$

Correspondingly, eqn. (25) becomes

$$
A_{i}{ }^{0}=\sum_{j \neq i} d_{i j} A_{j}{ }^{0}+\sum_{j} e_{i j} \frac{\partial A_{j}{ }^{0}}{\partial n^{0}}
$$

where $i, j \in B_{k}{ }^{0}$ and $B_{k}{ }^{0} \subset B=B_{1} \cup B_{2} \cup \ldots \cup B_{M}$. In this equation

$$
\begin{aligned}
& d_{i j}=\frac{1}{\pi} \int_{\Delta l_{j}} \frac{\cos \psi_{i j}^{0}}{r_{i j}} d l \\
& e_{i j}=-\frac{1}{\pi} \int_{\Delta l_{j}} \ln r_{i j} d l
\end{aligned}
$$

Approximating (26), we get

$$
\sum_{\in B_{m}} \Delta l_{j} \frac{\partial A_{j}}{\partial n}=-\mu_{m} I_{m}, m=1, \ldots, M
$$

All the elements, except for $b_{i i}$ and $e_{i i}$, are computed by using standard Gauss quadrature. An expression for the element $b_{i l}$ can be found in Salon et al. (1981), and $e_{i t}$ is evaluated analytically. 
Using the boundary conditions (i) and (ii), we get

$$
\begin{gathered}
A_{t}=A_{i}{ }^{0} \\
\frac{1}{\mu_{m}} \frac{\partial A_{i}}{\partial n}=-\frac{1}{\mu_{0}} \frac{\partial A_{i}{ }^{0}}{\partial n^{0}}
\end{gathered}
$$

We insert for $A_{i}{ }^{0}$ and $\partial A_{i}{ }^{0} \partial n^{0}$ from these equations in (36). On each element $\Delta l_{i}$ we then have two unknowns, namely the vector potential $A_{i}$ and its normal derivative $\partial A_{t} / \partial n$. We also have two equations for each element, one from the interior problem inside a conductor, eqn. (33), and one from the exterior problem, eqn. (36). The interior problem for a conductor involves unknowns only from the boundary of the conductor. The exterior problem on the other hand, couples unknowns from several conductors.

We define the unknowns as

$$
\left.\begin{array}{cc}
x_{i}=A_{i}, & i=1, \ldots, N \\
x_{N+t}=\partial A_{i} / \partial n, & i=1, \ldots, N \\
x_{2 N+m}=\phi_{m}, & m=1, \ldots, M
\end{array}\right\}
$$

Using (33) and (36) on each node on the boundary and eqn. (39) on each conductor, we get an equation system with $2 N+M$ unknowins.

$$
F \boldsymbol{x}=\boldsymbol{g}
$$

The solution $\boldsymbol{x}$ is found by standard Gaussian elimination. From $\boldsymbol{x}$ we can compute $E$ at the boundary (eqn. (9)), and the dissipated power $\boldsymbol{P}_{\boldsymbol{m}}$ within each conductor. After discretization, eqn. (31) becomes

$$
P_{m}=-\frac{\omega}{\mu_{m}} \operatorname{Re} \sum_{j \in B_{m}} i\left(A_{j}-\phi_{m}\right) \frac{\partial A_{j}^{*}}{\partial n} \Delta l_{j}, m=1, \ldots, M
$$

To find $A=A_{p}$ at a point $p$ inside conductor no. $m$, we use eqn. (20). After discretization, we get

$$
A_{p}=\left(1-\frac{1}{2} \sum_{j} a_{p j}\right) \phi_{m}+\frac{1}{2} \sum_{j} a_{p j} A_{j}+\frac{1}{2} \sum_{j} b_{p j} \frac{\partial A_{j}}{\partial n}
$$

where $p \in R_{m}$ and $j \in B_{m}$.

Special attention is needed if the point $p$ is near the boundary. The relative variations in the distance $r_{p j}$ can be significant for the nearest elements. When evaluating the integrals $a_{p j}$ and $b_{p j}$, we may also be fairly close to the singularity in $K_{\mathrm{o}}$ and $K_{1}$. The effective distance from the singularity depends on the ratio $r / \delta$ where $\delta$ is the skin depth. The skin depth is related to the constant $\alpha$ by the equation

$$
\alpha=\sqrt{2 i} i / \delta
$$

From Abramowitz and Stegun (1965) we have

$$
\begin{array}{ll}
K_{0}(\alpha r)=K_{0}(\sqrt{2 i} r / \delta) \rightarrow-\ln (\sqrt{2} r / \delta) & \text { as } r \rightarrow 0 \\
K_{1}(\alpha r)=K_{1}(\sqrt{2 i} r / \delta) \rightarrow \delta / r \sqrt{2 i} & \text { as } r \rightarrow 0
\end{array}
$$


We have separated out the singular terms and integrated them analytically for $r / \delta<h$ where $h$ is a suitable factor, for example $h=1$. The remaining integrands are then integrated by standard Gauss quadrature.

From $A_{p}$, we can find $E_{p}$ and $J_{p}$.

\section{Numerical results}

The sample problem, see Fig. 4, involves three conductors surrounded by a circular shielding. The conducting regions have skin depth $\delta=0.667 \mathrm{~cm}$ at an angular frequency $\omega=2 \pi \cdot 50 \mathrm{rad} / \mathrm{s}$. The total current flowing in each of the three conductors is $1 \mathrm{~A}$ with phases $0,2 \pi / 3$ and $4 \pi / 3$ respectively. The total current in the shielding is $0 \mathrm{~A}$.

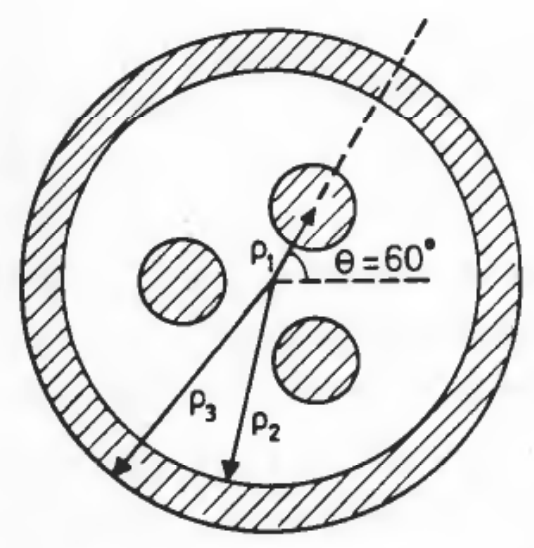

Figure 4. Sample problem where $\rho_{1}=2 \mathrm{~cm}, \rho_{2}=5 \mathrm{~cm}$, and $\rho_{3}=6 \mathrm{~cm}$. In the conducting regions, $\sigma=5 \cdot 7 \cdot 10^{7}(\Omega \mathrm{m})^{-1}, \mu=\mu_{0}=4 \pi \cdot 10^{-7} \mathrm{Hm}^{-1}$.

Using a finite difference method (FDM), we compute the dissipated power $P_{s}$ in the shielding as a sum over the volume and get $P_{s}=8 \cdot 26 \cdot 10^{-6} \mathrm{~W} / \mathrm{m}$. The solution domain is here $\rho<\rho_{\infty}=3 \rho_{3}$, and the boundary condition is given at $\rho_{\infty}$ as $\partial A / \partial n=\mu I / 2 \pi \rho_{\infty}=0$ because the total current is zero. The number of unknowns in the domain is 900 .

We approximate the three conductors with line currents and divide the boundary of the shielding into 64 elements ( 32 on each side). We compute $P_{s}$ as a sum along the boundary by using (44). The result is $P_{s}=8 \cdot 50 \cdot 10^{-6} \mathrm{~W} / \mathrm{m}$.

As a comparison, we let the three conductors have a radius of $1 \mathrm{~cm}$ and divide each of the three boundaries into 16 elements. Using BEM we get $P_{s}=8 \cdot 50 \cdot 10^{-6} \mathrm{~W} / \mathrm{m}$ while the dissipated power in each of the three conductors is $P_{c}=6 \cdot 35 \cdot 10^{-4} \mathrm{~W} / \mathrm{m}$.

We also compute $|E|$ along a cut in the shielding by using BEM. In this case $\theta=60^{\circ}$ and $\rho_{2}<\rho<\rho_{3}$, see Fig. 4. In the Table the results are compared with the finite difference solution. Here $r$ is the shortest distance from an internal point to the boundary, and $\Delta l$ is the length of an element. For the results given as BEM 1 , the singular terms in $K_{0}$ and $K_{1}$ are integrated analytically, where as in BEM 2 they are not. We see that the agreement between FDM and BEM 1 is very good, while the accuracy for BEM 2 is poor for points close to the boundary. 


\begin{tabular}{llllllll}
\hline$r / \delta$ & 0.00 & 0.15 & 0.45 & 0.75 & 0.45 & 0.15 & 0.00 \\
$r / \Delta l$ & 0.00 & 0.10 & 0.33 & 0.50 & 0.33 & 0.10 & 0.00 \\
FDM & & 1.088 & 0.923 & 0.827 & 0.772 & 0.737 & \\
BEM 1 & 1.21 & 1.074 & 0.918 & 0.826 & 0.772 & 0.735 & 0.724 \\
BEM 2 & 1.21 & 0.934 & 0.912 & 0.826 & 0.760 & 0.557 & 0.724 \\
\hline
\end{tabular}

The electric field $|E|\left(10^{-6} \mathrm{~V} / \mathrm{m}\right)$.

Figure 5 shows $|E|$ around the shielding for $\rho=\left(\rho_{2}+\rho_{3}\right) / 2$. For symmetry reasons, only computed values for $0<\theta<120^{\circ}$ are given. The results using BEM and FDM shows good agreement.

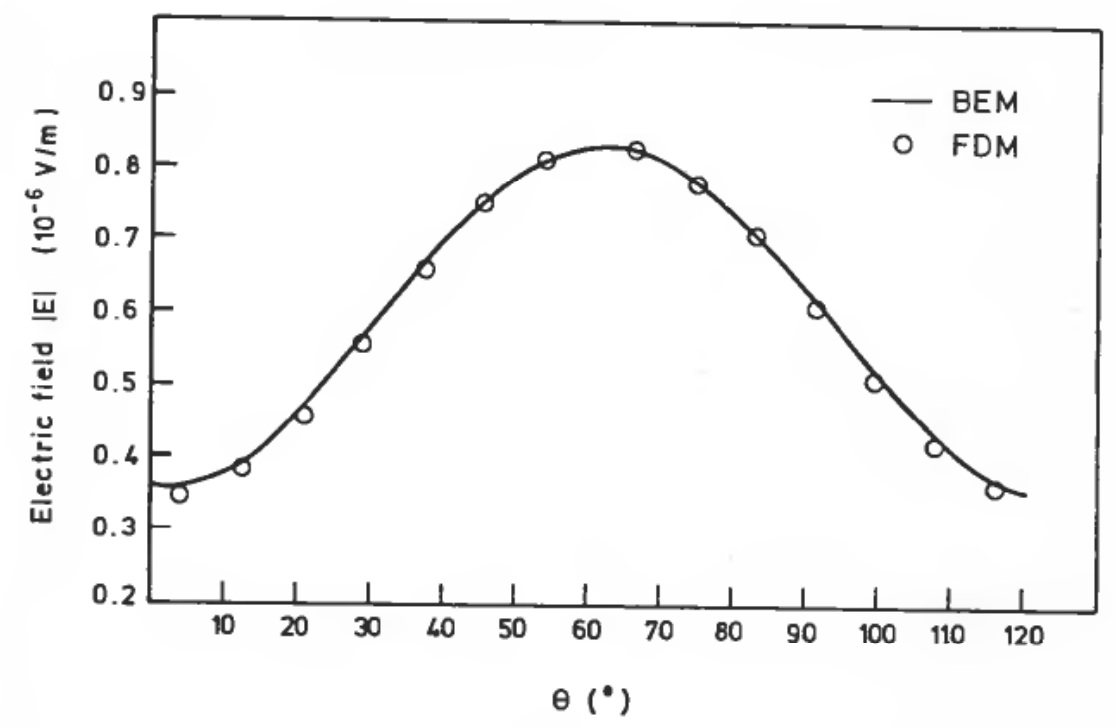

Figure 5.

\section{REFERENCES}

Abramowitz, M., and Stegun, I. A. (1965). Handbook of Mathematical Functions (Dover). ANDERSEN, O. W. (1977). Finite element solution of skin effect and eddy current problems. I.E.E.E. paper, A 77 616-6.

CARPENTER, C. J. (1977). Comparation of alternative formulations of 3-dimensional magneticfield and eddy-current problems at power frequencies. Proc. IEE, 124, 66-74.

Chari, M. V. K. (1973). Finite element solution of the eddy current problem in magnetic structures. I.E.E.E. paper, T 73 320-9.

EKRANN, S., and SiRA, T. (1982). A model for solving the Maxwell quasi-stationary equations in a three phase electric furnace. Modeling, Identification and Control, 3, 231-242.

SALON, S. J., SCHNEIDER, J. M., and UDA, S. (1981). Boundary element solutions to the eddy current problem. Proc. of the 3rd international seminar on boundary element methods (Springer Verlag), pp. 14-25. 